\title{
Erratum to: Serum HMGB1 concentrations at 4 weeks is a useful predictor of extreme poor prognosis for advanced hepatocellular carcinoma treated with sorafenib and hepatic arterial infusion chemotherapy
}

\author{
Kazuhiko Masuda $^{1}$ Atsushi Ono ${ }^{1,2,3,4} \cdot$ Hiroshi Aikata $^{1,2} \cdot$ Tomokazu Kawaoka $^{1,2}$. \\ C. Nelson Hayes ${ }^{1,2,3} \cdot$ Yuji Teraoka $^{1,2}$ - Kana Daijo ${ }^{1,2}$ - Yuki Nakamura-Inagaki ${ }^{1,2}$. \\ Kei Morio $^{1,2}$ - Hatsue Fujino ${ }^{1,2}$ - Hiromi Kan ${ }^{1,2}$. Takuro Uchida ${ }^{1,2} \cdot$ Keiichi Masaki $^{1,2}$. \\ Tomoki Kobayashi $^{1,2} \cdot$ Takashi Nakahara $^{1,2}$ - Grace Naswa Makokha ${ }^{1,2}$. \\ Yizhou Zhang ${ }^{1,2} \cdot$ Yuko Nagaoki $^{1,2} \cdot$ Daiki Miki ${ }^{2,3} \cdot$ Masataka Tsuge ${ }^{1,2}$. \\ Akira Hiramatsu $^{1,2} \cdot$ Michio Imamura ${ }^{1,2}$ Hiromi Abe-Chayama ${ }^{1,2,3}$. \\ Yoshiiku Kawakami $^{1,2} \cdot$ Hidenori Ochi $^{1,2,3} \cdot$ Kazuaki Chayama $^{1,2,3}$
}

Published online: 29 May 2017

(C) Japanese Society of Gastroenterology 2017

\section{Erratum to: J Gastroenterol \\ DOI 10.1007/s00535-017-1348-8}

The errors in the publication of the original article have been updated and the details are given below for your reading:
An article note "Kazuhiko Masuda and Atsushi Ono are the co-first authors" should be included.

The second sentence in the subheading "Results" under the "Abstract" section should read as "High HMGB1 at 4 weeks $(P=0.025)$, vascular invasion to the hepatic vein $(\mathrm{Vv}) \quad(P=0.009), \quad$ mRECIST
Kazuhiko Masuda and Atsushi Ono are the co-first authors.

The online version of the original article can be found under doi:10.1007/s00535-017-1348-8.

Kazuhiko Masuda

masu6743@hiroshima-u.ac.jp

Atsushi Ono

atsushi-o@hiroshima-u.ac.jp

Hiroshi Aikata

aikata@hiroshima-u.ac.jp

Tomokazu Kawaoka

kawaokatomo@hiroshima-u.ac.jp

C. Nelson Hayes

nelsonhayes@hiroshima-u.ac.jp

Yuji Teraoka

teraoka22@hiroshima-u.ac.jp

Kana Daijo

kanakana624@hiroshima-u.ac.jp
Yuki Nakamura-Inagaki ynakamur@hiroshima-u.ac.jp

Kei Morio

kmorio@hiroshima-u.ac.jp

Hatsue Fujino

fujino920@hiroshima-u.ac.jp

Hiromi Kan

hiromikan@hiroshima-u.ac.jp

Takuro Uchida

d136452@hiroshima-u.ac.jp

Keiichi Masaki

masakik8097@hiroshima-u.ac.jp

Tomoki Kobayashi

tomoki@hiroshima-u.ac.jp

Takashi Nakahara

nakahara@hiroshima-u.ac.jp

Grace Naswa Makokha

gnaswa@hiroshima-u.ac.jp 
$(P<0.0001)$ and Child-Pugh B $(P=0.004)$ were identified as independent predictors of poor overall survival in HAIC treatment."

The original article was corrected.

Yizhou Zhang

zhangyizhou10@yahoo.co

Yuko Nagaoki

nagaoki@hiroshima-u.ac.jp

Daiki Miki

daikimiki@hiroshima-u.ac.jp

Masataka Tsuge

tsuge@hiroshima-u.ac.jp

Akira Hiramatsu

akirah@hiroshima-u.ac.jp

Michio Imamura

mimamura@hiroshima-u.ac.jp

Hiromi Abe-Chayama

habe@hiroshima-u.ac.jp

Yoshiiku Kawakami

kamy4419@hiroshima-u.ac.jp

Hidenori Ochi

hochi@hiroshima-u.ac.jp

Kazuaki Chayama

chayama@hiroshima-u.ac.jp
1 Department of Gastroenterology and Metabolism, Applied Life Science, Institute of Biomedical and Health Science, Hiroshima University, 1-2-3 Kasumi, Minami-ku, Hiroshima 734-8551, Japan

2 Liver Research Project Center, Hiroshima University, Hiroshima, Japan

3 Laboratory for Digestive Diseases, Center for Genomic Medicine, The Institute of Physical and Chemical Research (RIKEN), Hiroshima, Japan

4 Division of Liver Diseases, Department of Medicine, Liver Cancer Program, Tisch Cancer Institute, Graduate School of Biomedical Sciences, Icahn School of Medicine at Mount Sinai, 1470 Madison Ave, Box 1123, New York, NY 10029, USA 